\title{
Mentored Patient-Oriented Research Career Development Award
}

National Cancer Institute

\section{Source}

National Cancer Institute. Mentored Patient-Oriented Research Career Development

Award. NCI Thesaurus. Code C19763.

To support the career development of investigators who have made a commitment to focus their research endeavors on patient-oriented research. This mechanism provides support for supervised study and research for clinically trained professionals who have the potential to develop into productive, clinical investigators focusing on patientoriented research. 